Artigo Original

\title{
Caracterização dos achados do processamento auditivo e fonológico em crianças com Doença Cerebrovascular-Isquêmica
}

\section{Characterisation of the auditory and phonologic process in children with Ischemic Cerebral Vascular Disease}

\author{
Cláudia Maria Sedrez Gonzaga Ronchi ${ }^{1}$, Leonardo Souza Oliveira' ${ }^{1}$ Simone Aparecida Capellini², \\ Sylvia Maria Ciasca $^{3}$, Maria Valeriana Leme de Moura-Ribeiro ${ }^{4}$
}

\section{RESUMO}

A criança acometida por Doença Cérebro Vascular (DCV) apresenta alterações de habilidades auditivas e fonológicas transitórias ou permanentes dependendo da época, do tipo e do local da lesão neurológica. Objetivo: Caracterizar os achados do processamento auditivo e fonológico em sujeitos com DCV-Isquêmica e relacionar os dados da avaliação do processamento auditivo e fonológico com os achados neurológico e de neuroimagem. Método: Participaram, deste estudo 5 sujeitos, sendo 4 meninos e uma menina na faixa etária de 7 a 13 anos de idade. Foram realizadas avaliações neurológica, de imagem, prova de consciência fonológica, prova de nomeação automática rápida. Resultados: Os sujeitos com DCV-I com insulto à direita e à esquerda apresentaram comprometimento em habilidade auditiva e em processamento fonológico, ocasionando lentidão quanto ao acesso e evocação do léxico mental, entretanto, ressaltamos que o comprometimento foi maior no sujeito com insulto à esquerda. Conclusão: Os achados deste estudo revelaram que quando há insulto em áreas neurológicas frontais, temporais e parietais em ambos os hemisférios, as habilidades referentes ao acesso ao léxico mental, ao uso da memória de trabalho e a consciência fonológica ficam prejudicadas, isto porque são dependentes do uso do processamento auditivo-verbal situado nestas regiões.

Unitermos: Acidente cerebrovascular, Percepção auditiva, Percepção da fala.

Citação: Ronchi CMSG, Capellini SA, Oliveira LS, Ciasca SM, Moura-Ribeiro MVL. Caracterização dos achados do processamento auditivo e fonológico em crianças com Doença Cerebrovascular-Isquêmica. Rev Neurociencias 2005; 13(2):061-066.

\section{SUMMARY}

Subject: The child with Cerebral Vascular Disease (CVD) presents auditory and phonological disorders that can be transitory or permanent depending on the period, type and localization of neurological lesion. Objective: Characterize the auditory and phonologic process findings in subjects with Ischemic CVD and connect the data of auditory and phonological evaluation with neurological and neuroimaging findings. Method: 5 subjects ( 4 boys and one girl) formed

Trabalho Realizado: Faculdade de Filosofia e Ciências da Universidade Estadual de Campinas - FCM/UNICAMP

1 - Fonoaudióloga e Neurologista Infantil. Aluna do Programa de Pós-Graduação em Ciências Médicas da Faculdade de Ciências Médicas - FCM/UNICAMP-Campinas - SP

2 - Fonoaudióloga. Doutora e Pós-Doutoranda em Ciências Médicas da Faculdade de Ciências Médicas - FCM/UNICAMPCampinas - SP. Docente do departamento de Fonoaudiologia da Faculdade de Filosofia e Ciências - FFC/UNESP-Marília- SP 3 - Neuropsicóloga. Professora Assistente da Disciplina de Neurologia Infantil da Faculdade de Ciências Médicas - FCM/UNICAMPCampinas - SP

4 - Neurologista Infantil. Professora Titular da Disciplina de Neurologia Infantil da Faculdade de Ciências Médicas - FCM/UNICAMPCampinas - SP

* Grupo de Pesquisa do CNPq "Anormalidades Neurovasculares na Infância"

Endereço para correspondência: Simone Aparecida Capellini

Rua Bartolomeu de Gusmão, 10-84, Jardim América, Bauru - SP. CEP: 17.017-326

Telefone de Contato: 14- 3227-2432 ou 14-9702-5470

E-mail: sacap@uol.com.br

Trabalho recebido em 04/04/05. Aprovado em 29/07/05 
Study Group, 7 to 13 years old. It has been done neurological evaluations, imaging, phonologic awareness test, and rapid automatic naming. Results: The subjects with Ischemic CVD with right and left damage present auditory and phonological disorder, causing slowness as access and recall of the mental lexicon, however, we emphasize that the major disorder was in the subjects with left damage. Conclusion: The findings of this study revealed that when there is damage in frontal, temporal, and parietal areas in both hemispheres, the abilities of access of the mental lexicon, use to working memory and phonological awareness were altered, because they are dependent of the verbal-auditory process located in this areas.

\section{Keywords: Cerebrovascular disease, Auditory perception, Speech perception.}

Citation: Ronchi CMSG, Capellini SA, Oliveira LS, Ciasca SM, Moura-Ribeiro MVL. Characterisation of the auditory and phonologic process in children with Ischemic Cerebral Vascular Disease. Rev Neurociencias 2005; 13(2):061-066.

\section{INTRODUÇÃO}

Dentre as afecções que podem comprometer o Sistema Nervosos Central (SNC), destaca-se a Doença Cérebro Vascular (DCV), termo utilizado para designar anormalidades do cérebro como resultado de processo patológico nos vasos sangüíneos.

Nas últimas duas décadas, os estudos sobre essa doença na infância auxiliado por recursos laboratoriais e técnicas não-invasivas de neuroimagem possibilitaram a ampliação dos conhecimentos pelos profissionais da área da saúde, particularmente, sobre a DCV em recém-nascidos a termo, em crianças e adolescentes. Segundo a literatura, a incidência da DCV na população infantil gira em torno de 2,52/100.000 a 7,91/100.000 habitantes/ano ${ }^{1,2}$.

Embora na literatura, se verifique crescente interesse pela investigação da DCV na infância, ainda é restrito o número de trabalhos que enfoquem o impacto desta anormalidade na aquisição e desenvolvimento da linguagem da criança. Tomando por base que a lesão no SNC, decorrente da DCV, pode ocorrer em áreas focais ou difusas alterando as funções neuronais, as habilidades da linguagem podem encontrar-se alteradas de forma transitória ou permanente dependendo da época, do tipo e do local da lesão neurológica.

Para a ocorrência do processamento lingüístico adequado é necessário que as habilidades auditivas estejam íntegras, porque desta forma o sinal acústico da fala será codificado, decodificado e integrado garantindo a criança o reconhecimento de padrões fonológicos presentes na linguagem oral ${ }^{3}$.

Tanto no comportamento auditivo mais elementar como nas tarefas de linguagem receptiva, as habilidades de processamento auditivo e as habilidades de processamento cognitivo estão intimamente interconectadas, não podendo ser facilmente separadas em seus diferentes componentes ${ }^{4}$.

Com base no exposto acima, este estudo tem por objetivos: 1) caracterizar os achados da avaliação do processamento auditivo e fonológico de crianças com DCV Isquêmica (DCV-I) e 2) relacionar os dados da avaliação do processamento auditivo e fonológico com os achados neurológico e de neuroimagem.

\section{MÉTODOS}

Participaram deste estudo 5 sujeitos, com idade entre 7 e 13 anos, (4 meninos e uma menina), nascidos de termo, com diagnóstico comprovado por imagem de lesão vascular cerebral unilateral. Esses sujeitos foram atendidos e orientados a partir da fase aguda da DCV na enfermaria de Pediatria do Hospital de Clínicas Faculdade de Ciências Médicas-UNICAMP.

Esses sujeitos foram submetidos a avaliação neurológica; tomografia computadorizada (CT); Ressonância Magnética (RM); Avaliação do processamento auditivo Teste de Dissílabos Alternados em Português - Staggered Spondaic Word test - SSW5,6; Teste dicótico consoante voga/7 e Teste de escuta dicótica com dígitos ${ }^{8}$; Avaliação do processamento fonológico (Prova da Consciência Fonológica- $P C F)^{9}$ e Prova de Nomeação Automática Rápida - RAN ${ }^{10}$. Os procedimentos aplicados nos sujeitos deste estudo são normatizados para a faixa etária dos mesmos.

Os procedimentos de processamento auditivo foram realizados no Ambulatório de Distúrbios da Comunicação no Laboratório de Audiologia do Departamento de Otorrinolaringologia da FCM UNICAMP. Os resultados da avaliação audiológica revelaram-se dentro dos padrões de normalidade, o que permitiu a realização das provas de processamento auditivo e fonológica.

O trabalho foi aprovado pelo Comitê de Ética em Pesquisa da FCM- UNICAMP, segundo as determinações do Conselho Nacional de Saúde (Resolução 196/96), sob o n²78/2001. O Termo de Consentimento Livre e Esclarecido foi assinado pelos responsáveis antes do início das avaliações.

\section{RESULTADOS}

Os dados referentes à identificação dos sujeitos, sexo, idade, local da lesão e etiologia da DCV-I estão descritos na Tabela 1. Os sujeitos S1, S3, S4 e S5 apresentam lesão cortical/subcortical, o sujeito S2 possui lesão apenas cortical. Os sujeitos S1, S3, S4 foram acometidas por lesão fronto-temporal e o sujeito S2 teve comprometimento parietal.

$\mathrm{Na}$ análise da Tabela 1 verificamos que 4 sujeitos apresentaram comprometimento do hemisfério direito e 


\begin{tabular}{|c|c|c|c|c|c|c|c|c|c|}
\hline Sujeitos & Hem. & Cor & Sexo & Idade* & Idade** & Local & TC-RM & $\begin{array}{c}\text { Artéria } \\
\text { envolvida }\end{array}$ & Etiologia \\
\hline S1 & $\mathrm{E}$ & $\mathrm{Br}$ & $\mathrm{M}$ & $8 \mathrm{a} 3 \mathrm{~m}$ & $4 \mathrm{a} 10 \mathrm{~m}$ & C-SC & FTP & ACM-RP & Trauma \\
\hline S2 & $\mathrm{D}$ & $\mathrm{Br}$ & $\mathrm{M}$ & $12 \mathrm{a} 4 \mathrm{~m}$ & $4 \mathrm{a} 11 \mathrm{~m}$ & $\mathrm{C}$ & $\mathrm{P}$ & ACM & Vasculite \\
\hline S3 & $\mathrm{D}$ & $\mathrm{Br}$ & $\mathrm{M}$ & $8 \mathrm{a} 1 \mathrm{~m}$ & $7 \mathrm{a}$ & C-SC & FTP & ACM & Arterite \\
\hline S4 & $\mathrm{D}$ & $\mathrm{Br}$ & $\mathrm{F}$ & $12 \mathrm{a} 4 \mathrm{~m}$ & $4 \mathrm{~m}$ & C-SC & FPT & RP-ACM & Sem etiologia \\
\hline S5 & $\mathrm{D}$ & $\mathrm{n} \mathrm{Br}$ & $\mathrm{M}$ & $12 \mathrm{a} 3 \mathrm{~m}$ & $3 \mathrm{~m}$ & C-SC & FP & ACM-RP & Sem etiologia \\
\hline
\end{tabular}

Legenda: Hem., hemisfério lesionado; E, esquerdo; D, direito; $\mathrm{Br}$, branco; ñBr, não branco; M, masculino; F, feminino; a, anos; m, meses; C, cortical; SC, subcortical; Fr, frontal; T, temporal; P, parietal; *, idade na avaliação; **, idade no momento da lesão; TC, tomografia computadorizada; RM, ressonância magnética; $\mathrm{ACM}$, artéria cerebral média e RP, ramos perfurantes.

Tabela 1. Doença Cérebro-vascular Isquêmica - Dados de identificação dos sujeitos do estudo.

um sujeito com comprometimento do hemisfério esquerdo, sendo o envolvimento vascular agudo no território da artéria cerebral média, com ou sem lesão de ramos perfurantes.

Na Tabela 2 registramos o desempenho em habilidade do processamento auditivo dos sujeitos apresentando o percentual de erros no teste Staggered Spondaic Word -SSW e no teste de escuta dicótica com dígitos (DD).

\begin{tabular}{|c|c|c|c|c|c|c|}
\hline No & $\begin{array}{c}\text { SSW } \\
\% \text { de } \\
\text { erros } \\
\text { DNC }\end{array}$ & DC & EC & ENC & $\begin{array}{c}\text { DD } \\
\% \text { de } \\
\text { erros } \\
\text { OD }\end{array}$ & OE \\
\hline 1 & 27,5 & 97,5 & 17,5 & 7,5 & 81,3 & 0,0 \\
\hline 2 & 5,0 & 15,0 & 22,5 & 0,0 & 9,0 & 2,5 \\
\hline 3 & 5,0 & 20,0 & 42,5 & 5,0 & 4,0 & 22,5 \\
\hline 4 & 10,0 & 42,5 & 17,5 & 7,5 & 72,0 & 17,5 \\
\hline 5 & 0,0 & 10,0 & 15,0 & 0,0 & 12,5 & 87,5 \\
\hline
\end{tabular}

Legenda: SSW: Staggered Spondaic Word, DNC, direita não competitiva; DC, direita competitiva; EC, esquerda competitiva; ENC, esquerda não competitiva, Teste de escuta dicótica com dígitos: $\mathrm{OD}$, orelha direita; $\mathrm{OE}$ orelha esquerda.

Tabela 2. Doença Cérebro-vascular Isquêmica - Distribuição do percentual de erros dos sujeitos no SSW.
$\mathrm{Na}$ análise dos dados constatamos percentual de erros muito alterado do sujeito S1 na condição direita competitiva; o sujeito $\mathrm{S} 4$, maior número de erros na orelha ipsilateral à lesão; os demais sujeitos obtiveram melhor desempenho na orelha ipsilateral a lesão. Evidenciamos no teste de dicótico de dígitos que o sujeito $\mathrm{S} 1$ apresentou $81,3 \%$ de erros na orelha contra lateral a lesão; o sujeito S3, 22,5\% erros na orelha contra lateral à lesão; o sujeito S4 e S5, alteração para as duas orelhas, e o sujeito S2 não apresentou alteração nas duas orelhas.

O desempenho dos sujeitos no teste consoante vogal referente aos acertos e erros nas três etapas, atenção livre, atenção direita e atenção esquerda está na Tabela 3.

Na análise da Tabela 3 observamos que o sujeito S1 obteve vantagem da orelha esquerda, ou seja, ipsilateral à lesão; os sujeitos S2, S3 e S5 com lesão em hemisfério direito apresentaram vantagem da orelha ipsilateral à lesão. O sujeito S4 apresentou desempenho inferior para a orelha ipsilateral à lesão.

A Tabela 4 apresenta o desempenho dos sujeitos na prova de consciência fonológica PCF.

$\mathrm{Na}$ análise da Tabela 4 constatamos desempenho inferior do sujeito S1 em relação aos demais sujeitos,

\begin{tabular}{|c|c|c|c|c|c|c|c|c|c|}
\hline \multirow{3}{*}{ Sujeitos } & \multicolumn{9}{|c|}{$\begin{array}{c}\text { Consoante } \\
\text { Vogal }\end{array}$} \\
\hline & $\begin{array}{l}\text { Atenção } \\
\text { livre }\end{array}$ & & & $\begin{array}{c}\text { Atenção } \\
\text { direita }\end{array}$ & & & $\begin{array}{l}\text { Atenção } \\
\text { esquerda }\end{array}$ & & \\
\hline & OD & OE & Erros & OD & OE & erros & OD & OE & Erros \\
\hline S1 & 2 & 21 & 2 & 1 & 19 & 4 & 1 & 16 & 7 \\
\hline $\mathrm{S} 2$ & 12 & 5 & 7 & 8 & 9 & 7 & 11 & 7 & 6 \\
\hline S3 & 18 & 2 & 4 & 20 & 1 & 3 & 12 & 1 & 11 \\
\hline S4 & 4 & 12 & 8 & 5 & 14 & 5 & 9 & 6 & 9 \\
\hline S5 & 12 & 6 & 6 & 16 & 4 & 4 & 15 & 5 & 4 \\
\hline
\end{tabular}

Legenda: OD, orelha direita; OE, orelha esquerda.

Tabela 3. Doença Cérebro-vascular Isquêmica - Distribuição dos acertos e erros dos sujeitos no teste consoante vogal, nas 3 etapas: atenção livre, atenção direita e atenção esquerda. 


\begin{tabular}{|c|c|c|c|c|c|c|c|c|c|c|c|}
\hline \multirow{2}{*}{ Sujeitos } & \multicolumn{11}{|c|}{ PCF } \\
\hline & SiS & SiF & Rima & Alit. & $\mathrm{Ses}$ & SeF & ManS & ManF & TrS & TrF & Total \\
\hline S1 & 4 & 1 & 2 & 2 & 3 & 0 & 1 & 1 & 0 & 0 & 14 \\
\hline S2 & 4 & 3 & 4 & 4 & 4 & 4 & 4 & 4 & 4 & 2 & 37 \\
\hline S3 & 4 & 3 & 4 & 4 & 4 & 3 & 4 & 4 & 4 & 1 & 35 \\
\hline S4 & 4 & 4 & 4 & 4 & 4 & 4 & 4 & 4 & 4 & 1 & 37 \\
\hline S5 & 4 & 4 & 4 & 3 & 4 & 4 & 4 & 4 & 4 & 2 & 37 \\
\hline
\end{tabular}

Legenda: SiS, síntese silábica; SiF, síntese fonêmica; Alit, aliteração; SeS, segmentação silábica; SeF, segmentação fonêmica; ManS, manipulação silábica; ManF, manipulação fonêmica; TrS, transposição silábica; TrF, transposição fonêmica.

Tabela 4. Doença Cérebro-vascular Isquêmica - Distribuição do desempenho dos sujeitos na PCF.

com exceção da tarefa de síntese silábica. Os outros 4 sujeitos apresentaram maior dificuldade na transposição fonêmica, tarefa que exige raciocínio e planejamento para modificar os fonemas de posição para formação de nova palavra. O sujeito S2 apresentou desempenho dentro do normal para escolaridade e faixa etária.

$\mathrm{Na}$ Tabela 5 apresentamos o desempenho dos sujeitos na prova de Nomeação Automática Rápida.

Na análise da Tabela 5 evidenciamos maior lentidão nos 4 subtestes do sujeito $\mathrm{S} 1$ em relação aos demais sujeitos.

\begin{tabular}{|c|c|c|c|c|}
\hline \multirow{2}{*}{ Sujeitos } & \multicolumn{4}{|c|}{ Tempo } \\
\cline { 2 - 5 } & Cores & Letras & Números & Objetos \\
\hline 1 & $1: 55$ & $1: 45$ & $1: 50$ & $2: 05$ \\
\hline 2 & $0: 44$ & $0: 24$ & $0: 24$ & $0: 46$ \\
\hline 3 & $0: 57$ & $0: 33$ & $0: 39$ & $0: 57$ \\
\hline 4 & $0: 55$ & $0: 22$ & $0: 28$ & $1: 03$ \\
\hline 5 & $0: 52$ & $0: 38$ & $0: 32$ & $1: 01$ \\
\hline
\end{tabular}

Tabela 5. Doença Cérebro-vascular Isquêmica - Distribuição dos valores em minutos e segundos dos sujeitos na prova da RAN.

\section{DISCUSSÃO}

Na análise do desempenho dos sujeitos deste estudo relacionando as habilidades auditivas com o processamento fonológico verificamos que o sujeito S1 apresenta memória auditiva comprometida com padrão de resposta tipo A, identificada pela testagem com o SSW, revelando alteração de processamento auditivo do tipo decodificação e integração. Estas alterações de processamento estão provavelmente relacionadas a localização da lesão em região têmporo-parietal à esquerda envolvendo o giro temporal médio e superior, região esta que contém as conexões necessárias para a codificação, decodificação e integração do estímulo auditivo; este comprometimento ocasiona alterações no uso de estratégias de evocação e recuperação de padrões fonêmicos e silábicos para formação de palavras ou acesso ao léxico mental evidenciado objetivamente em provas de consciência fonológica e de memória de trabalho, como a PCF e a RAN. Ressaltamos ainda, que este sujeito 1 apresentou alteração significante em consoante-vogal e dicótico de dígitos, o que compromete o uso de processos atencionais e perceptivos para formação dos elementos acústicos-fonêmicos da palavra.

Na análise realizada com os sujeitos S2, S3 e S4, verificamos que apesar do insulto ter acometido o hemisfério direito, o desempenho em relação a memória auditiva foi semelhante ao S1, ou seja, com comprometimento e padrão de resposta tipo A, identificada pelo testagem com o SSW, revelando alteração de processamento auditivo do tipo decodificação e integração. Entretanto, também foi evidenciado alteração referente ao efeito auditivo, o que justificaria o comprometimento de memória destes sujeitos, uma vez que esta análise qualitativa se refere a categorização de perda gradual de memória, em nível de processamento auditivo. Tal alteração provavelmente se deve ao fato destes pacientes apresentarem lesão em lobo parietal, como o sujeito S2 e em lobos fronto-têmporo-parietal, como os sujeitos S3 e S4.

O perfil auditivo e fonológico destes sujeitos está relacionado com a dificuldade em organizar e planejar a ordem para composição e recomposição de palavras conforme evidenciada nos testes de processamento auditivo consoante-vogal, dicótico de dígitos, no subteste de transposição fonêmica da PCF e a lentidão no acesso ao léxico mental demonstrada em cores e objetos da RAN.

Quando a criança apresenta lentidão na produção ou evocação da palavra é indicativo de atraso no desenvolvimento da fala e leitura, uma vez que isto decorre de dificuldade de associação do código visual com o código fonológico ${ }^{11-13}$

Com relação ao sujeito S5 pudemos verificar que como o mesmo apresentou insulto neurológico em idade mais precoce que os demais, não foi identificado alterações referente a decodificação ou integração de estímulos auditivos no SSW, porém como sua lesão acometeu território fronto-parietal direito, foi evidenciado dificuldade quanto a realização dos testes de processamento auditivo referentes a consoante-vogal e o dicótico de dígitos, revelando assim dificuldade quan- 
to ao uso de processos atencionais e perceptivos para formação dos elementos acústicos-fonêmicos da palavra evidenciada no subteste de transposição fonêmica da PCF e no uso da memória de trabalho fonológica.

A reorganização neurológica pós-insulto no SNC, é mais eficaz em crianças com idade precoce, isto porque as chances de plasticidade para a linguagem são maiores e a ocorrência de distúrbios em funções específicas cognitivo-lingüísticas são menores, fato confirmado no sujeito S5 que teve acometimento vascular agudo identificado aos 3 meses de idade ${ }^{14,15}$

Neste estudo evidenciamos que a consciência fonológica está diretamente relacionada com a codificação fonológica desencadeada pelas habilidades auditivas, necessárias para a realização da correspondência visual-auditiva exigida tanto na linguagem oral como escrita ${ }^{12,16,17}$.

A relação evidenciada entre o processamento auditivo e o processamento fonológico da informação está no fato da decodificação da comunicação oral ter seu início com a decifração do sistema de sons da cadeia da palavra no lobo temporal do hemisfério esquerdo. $\mathrm{Na}$ medida que a informação vai entrando, vai sendo arquivada seqüencialmente com marcação de tempo e de ordem, permitindo identificar e evocar os elementos que estão entrando. A palavra é reconhecida como tal porque se registra união entre o produto da decodificação e uma memória prévia, denominada lexical18-19.

Entretanto, apesar de conhecermos e reconhecermos que o processo de decodificação da composição fonêmica da linguagem está localizada no lobo temporal do hemisfério esquerdo, ressaltamos que a análise da estrutura fonológica da linguagem oral, que envolve diretamente a percepção do som, é o mais alto estágio em termos de função cortical, e deve ser entendida como parte de um sistema funcional complexo, que ocorre por meio da participação de grupos de estruturais cerebrais operando em concerto, com cada qual contribuindo para a organização desse sistema funcional ${ }^{20}$.

Sendo assim, existe o entendimento de que o hemisfério direito é parte atuante deste processamento da linguagem quanto a tarefas de análise e julgamento21,22.

\section{REFERÊNCIAS BIBLIOGRÁFICAS}

1. Moura-Ribeiro MVL, Ciasca SM. Afecções vasculares cerebrais na infância-condutas. In: Moura-Ribeiro MV, Ferreira LS. Condutas em neurologia infantil. Rio de Jareiro-RJ: Revinter; 2004.

2. Giroud M, Lemesle M, Gouyon JB, Nivelon JL, Milan C, Dumas R. Cerebrovascular disease in children under 16 year of age in the city of Dijon, France: a study of incidence
A tarefa de decisão lexical, freqüentemente utilizada para comparar modelos de processamento hemisférico, tem revelado que o modelo de acesso direto enfatiza a especialização hemisférica relativa, supondo que cada hemisfério é capaz de processar os estímulos apresentados a ele diretamente, embora com níveis desiguais de eficiência. Alternativamente, retransmissão calosa se refere à transmissão calosa de um estímulo quando projetado ao hemisfério não-especializado. Assim, apesar do hemisfério esquerdo ser predominante para aquisição da linguagem oral e escrita, a integração dos hemisférios cerebrais, tanto esquerdo como direito, são interdependentes para a identificação perceptual das palavras ${ }^{23}$

Com relação a esta interdependência hemisférica, para a habilidade auditiva, os dados deste estudo corroboram os achados de Divenyi e Robinson ${ }^{24}$ que confirmaram a prevalência do hemisfério direito para o processamento da informação espectral da palavra.

\section{CONCLUSÃO}

Com base nos resultados deste estudo, concluímos que:

- Os sujeitos com DCV-I com insulto à direita e à esquerda apresentam comprometimento em habilidade auditiva e em processamento fonológico, apresentando como conseqüência lentidão quanto ao acesso e evocação do léxico mental, entretanto, ressaltamos que o comprometimento foi maior no sujeito com insulto à esquerda;

- Há relação entre os achados do processamento auditivo, do processamento fonológico com os achados neurológicos e de imagem evidenciando que quando há insulto em áreas neurológicas frontais, temporais e parietais em ambos os hemisférios, as habilidades referentes ao acesso ao léxico mental, o uso da memória de trabalho e a consciência fonológica ficam prejudicadas, isto porque o processamento temporal da informação relacionados às habilidades auditivo-verbais parecem, conforme evidenciado neste estudo, estar situadas nestas regiões. and clinical features from 1985 to 1993. J Clin Epidemiol 1995; 48:1343-1348.

3. Cervera-Mérida JF, YguaL-Fernandez A. Intervención logopédica en los trastornos fonológicos desde el paradigma psicolingüístico del procesamiento del habla. Rev Neurol 2003; 36:39-53.

4. Momensohn-Santos TM, Branco-Barreiro FCA. Avaliação e intervenção fonoaudiológica no transtorno de proces- 
samento auditivo. In: Ferreira LP, Befi-Lopes DM, Limongi SCO. Tratado de fonoaudiologia. São Paulo: Roca; 2004.

5. Katz J, Basil RA, Smith JM. A staggered spondaic word test for detecting central auditory lesion. Annals at Congress Otology, Rhinology and Laringology, 1963; 72: 908-917.

6. Borges ACLC. Dissílabos alternados - SSW. In: Pereira LD, Schochat E. Processamento auditivo central: Manual de avaliação. São Paulo: Lovise, 1997.

7. Pinto ALPG. Atenção seletiva para estímulos verbais: teste de escuta dicótica. São Paulo. (Monografia de Especialização), São Paulo: Universidade Federal de São Paulo Escola Paulista de Medicina; 1991.

8. Santos MFC, Pereira LD. Escuta com dígitos. In: Pereira. LD, Schochat E. Processamento auditivo central: Manual de avaliação. São Paulo: Lovise; 1997.

9. Capovilla AGS, Capovilla FC. Prova de consciência fonológica: desenvolvimento de dez habilidades da pré-escola à segunda série. Tem sobre desenvolv 1998; 7: 14-20.

10. Denckla MB, Rudel R. Rapid automatized naming of pictured objects, colors, letters and numbers by normal children. Cortex 1974; 10:186-202.

11. Ellis AW. Leitura, escrita e dislexia: uma análise cognitiva. 2.ed. Porto Alegre-RS: Artes Médicas; 1995.

12. Ferreira TL, Capellini SA, Ciasca SM, Tonelotto JMF. Desempenho de escolares leitores proficientes no teste de nomeação automática rápida (RAN). Tem sobre desenvolv, 2003; 12:26-32.

13. Miranda-Casas A, Baixauli-Ferrer I, Soriano M, Presentación-Herrero MJ. Cuestiones pendientes en la investigación sobre dificultades del acceso al léxico: una visión de futuro. Rev Neurol 2003; 36: 20-28.

14. Chugani HT. Neuroimaging of developmental nonlinearity and developmental pathologies. Academic Press Inc; 1997.
15. Kandel ER, Schwartz JH, Jessell TM. Fundamentos da neurociência e do comportamento. Rio de Janeiro: Prentice-Hall do Brasil; 2000.

16. Cardoso-Martins C, Pennington BF. Qual é a contribuição da nomeação seriada rápida para a habilidade de leitura e escrita?: Evidência de criança e adolescente com e sem dificuldades de leitura. Psicol Refl Crít 2001; 14: 387-397.

17. Capellini SA Eficácia do programa de remediação fonológica em escolares com distúrbio específico de leitura e distúrbio de aprendizagem. (Tese de Doutorado), Campinas: Universidade Estadual de Campinas; 2001.

18. Luria AR. Pensamento e linguagem. Porto Alegre- RS: Artes Médicas; 1986

19. Castro-Caldas A. A Herança de Franz Joseph Gall: o cérebro a serviço do comportamento humano. Lisboa: McGRawHill; 2000.

20. Luria AR. Fundamentos de neuropsicologia. São Paulo: Editora da Universidade de São Paulo; 1984.

21. Ardila A. Participación de los lóbulos frontales en el lenguaje. In: Mansur LL, Rodrigues N. Temas em neurolingüística. Editor: Mansur \& Rodrigues. São Paulo: Tec Art; 1993.

22. Newman SD, Just MA, Mason R. Compreendendo o texto com o lado direito do cérebro: o que os estudos de neuroimagem cerebral têm a dizer. In: Rodrigues $\mathrm{C}$, Tomitch LMB. Linguagem e cérebro humano: contribuições multidisciplinares. Porto Alegre: Artes Médicas; 2004.

23. Waldie KE. O papel do hemisfério direito no desenvolvimento normal e prejudicado da leitura. In: Rodrigues C, Tomitch LMB. Linguagem e cérebro humano: contribuições multidisciplinares. Porto Alegre: Artes Médicas; 2004.

24. Divenyi PL, Robinson AJ. Nonlinguistic auditory capabilities in: aphasia. Brain Lang 1989; 37: 290-326. 